\title{
TEACHERS AND STUDENTS' DIFFICULTY AND COPING STRATEGY TOWARDS TECHNOLOGY AND LIVELIHOOD EDUCATION PROGRAM
}

*Aljaven Bruce C. Laganhon

Paper Received: 09.03.2020 / Paper Accepted: 29.05.2020 / Paper Published: 02.06.2020

Corresponding Author: Aljaven Bruce C. Laganhon; Email: aljaven25bruce@ gmail.com; doi:10.46360/globus.xxxxxxxx

\section{Abstract}

This study sought to determine the teachers and students' difficulty and coping strategy towards Technology and Livelihood Education Program of Domingo Lacson National High School, Bacolod City during the School Year 20162017.Respondents were 26 teachers and 313 students. It employed the descriptive research design and utilized self-made questionnaires. Specifically, it identified the profile of the respondents in terms of age, sex, length of service, educational attainment, and subject taught (for teachers) and age, sex, year level, and specialization (for students).The level of difficulty of teachers was moderate on physical facilities and assessment tools while high on instructional materials and competencies. Students were low on physical facilities and competencies while moderate on instructional materials and assessment tools. The extent of coping strategy of teachers was moderate on physical facilities and instructional materials while great on assessment tools and competencies. Students were moderate on physical facilities, assessment tools, and competencies while low on instructional materials. Significant difference exists in the level of difficulty of teachers on assessment tools when grouped according to age and on physical facilities when grouped according to subject taught. Significant difference exists in the level of difficulty of students on instructional materials and assessment tools when grouped according to age and on physical facilities and assessment tools when grouped according to specialization. Significant difference exists in the extent of coping strategy of teachers on instructional materials and assessment tools when grouped according to age and on physical facilities when grouped according to subject taught. Significant difference exists in the extent of coping strategy of students on instructional materials, assessment tools, and competencies when grouped according to year level and in all areas when grouped according to specialization.

Keywords: Teachers, Students, Difficulty, Coping Strategies, Technology and Livelihood Education Program.

\section{Introduction}

Teachers and students' difficulty and coping strategy towards Technology and Livelihood Education Program are essential dimensions to consider as they have significant impacts on the teaching and learning process itself.

However, at Domingo Lacson National High School, both teachers and students have been facing enormous difficulties in relation to teaching and learning TLE courses. Some of the teachers are forced to teach the subject even without formal background and training. These often resulted to poor evaluation. Likewise, the instructional materials and equipment are not sufficient due to lack of funds. In the same manner, due to limited areas offered by these schools, students are forced to get the mandatory TLE subjects.

Hence, to cope with these difficulties, teachers are forced to subject themselves for training and secure instructional materials and equipment at their own expense. Students, likewise, have to exert more time and effort in understanding and memorizing a whole lot of concepts on TLE-related courses.

It is on this premise that the researcher, as TLE teacher, has been motivated to determine the teachers and students' difficulty and coping strategy towards the Technology and Livelihood Education Program of Domingo Lacson National High School, Bacolod City during the School Year 2016-2017.

\section{Objectives of the Study}

This study sought to answer the following questions:

1. What is the profile of the teacher respondents in terms of the following: age, sex, length of service, educational qualification, and subject taught?

2. What is the profile of the student-respondents in terms of the following: age, sex, year level, and specialization?

3. What is the level of difficulty of the teachers and students when variables are taken as a whole?

4. What is the extent of the coping strategy of the teachers and students when variables are taken as a whole?

*Carlos Hilado Memorial State College, Talisay City, Negros Occidental, Philippines.

Email: aljaven25bruce@gmail.com 
5. Is there a significant difference in the level of difficulty of the teachers and students when grouped according to variables?

6. Is there a significant difference in the extent of coping strategy of the teachers and students when grouped according to variables?

\section{Methodology}

Respondents were the twenty-two (26) teachers and three hundred and thirteen (313) students. In determining the number of student respondents, the researcher resorted to sample survey with the use of stratified sampling technique.

The study employed the descriptive research design. Furthermore, this study utilized self-made questionnaires, that is, one for the level of difficulty of teachers and students and one for the extent of coping strategy of teachers and students. Specifically, Part I of the questionnaire identified the demographic profile of the respondents in terms of age, sex, length of service, educational attainment, and subject taught (for teachers) and age, sex, year level, and specialization (for students). While Part 2 contained the survey proper that consisted of 40 items on the level of difficulty and extent of coping strategy. Each dimension had four (4) areas, namely, physical facilities, instructional materials, assessment tools, and competencies. Each area had ten (10) items or indicators.

The data were analyzed in relation to the research objectives:

Problems 1 and 2 employed the frequency count and percentage in determining the profile of the respondents.

Problems 3 and 4 employed the mean to determine the respondents' level of difficulty and extent of coping strategy towards TLE program.

Problems 5 and 6 employed the t-test to determine whether or not significant difference existed in the levels of difficulty and extents of coping strategy towards the TLE program when respondents are grouped according to age, sex, length of service, year level, and educational qualification while ANOVA was used to determine whether or not significant difference existed when respondents are grouped according to subject taught and specialization.

\section{Findings}

\section{Table 1: Profile of Teacher Respondents}

\begin{tabular}{llll}
\hline Variables & Categories & F & $\%$ \\
\hline \multirow{3}{*}{ Age } & Younger (26 to 39 years old) & 13 & 50.0 \\
& Older (40 to 59 years old) & 13 & 50.0 \\
Sex & Male & 13 & 50.0 \\
& Female & 13 & 50.0 \\
Length of Service & Shorter (1 to 11 years) & & \\
& Longer (12 to 37 years) & 13 & 50.0 \\
Educational Qualification & Machelor's Degree & 13 & 50.0 \\
& Master's Degree & 16 & \\
& & 10 & 38.5 \\
& Agriculture & & \\
& Carpentry & 4 & 15.4 \\
& Drafting & 3 & 11.5 \\
Subject Taught & Dressmaking & 3 & 11.5 \\
& HE & 3 & 11.5 \\
& ICT & 4 & 15.4 \\
& Massage & 3 & 11.5 \\
& SMAW & 3 & 11.5 \\
& & 3 & 11.5 \\
& & & \\
\hline
\end{tabular}

The teacher respondents were evenly distributed when considered by age, sex, and length of service. When segregated by educational qualification, the teachers with bachelor's degree constituted more than half of the total number of respondents. When considered by subject taught, each group was also evenly distributed except 
those teaching in agriculture and Home Economics/Home Nursing which constituted a little bit more than the rest of the groups.

Table 2: Profile of Student Respondents

\begin{tabular}{llll}
\hline Variables & Categories & f & \% \\
\hline \multirow{3}{*}{ Age } & Younger (14 to 15 years old) & 136 & 43.5 \\
& Older (16 to 21 years old) & 177 & 56.5 \\
Sex & Male & & \\
& Female & 155 & 49.5 \\
& Total & 158 & 50.5 \\
Year Level & Grade 9 & 313 & 100.0 \\
& Grade 10 & 167 & 53.4 \\
& Agriculture & 146 & 46.6 \\
& Carpentry & & \\
& Drafting & 40 & 12.8 \\
Specialization & Dressmaking & 39 & 12.5 \\
& HE/Home Nursing & 39 & 12.5 \\
& ICT & 39 & 12.5 \\
& Massage & 40 & 12.8 \\
& SMAW & 39 & 12.5 \\
& & 39 & 12.5 \\
& & 38 & 12.1 \\
\hline
\end{tabular}

On the other hand, the older group of students dominated its counterpart group. When considered by sex, the female group comprised a little more than the male ones. Segregated by year level, the grade 9 students comprised more than its counterpart. When considered by area of specialization, those taking up carpentry, drafting, dressmaking, Information Communications Technology, and massage were evenly distributed. The group with the least number of respondents came from those taking up Shielded Metal Arc Welding (SMAW).

Table 3.1: Level of Difficulty of Teachers When Variables are taken as a Whole

\begin{tabular}{|c|c|c|c|c|c|c|c|c|c|}
\hline Variable & Category & $\begin{array}{l}\text { Physi } \\
\text { Facilit }\end{array}$ & & $\begin{array}{l}\text { Instruc } \\
\text { Materi }\end{array}$ & & $\begin{array}{l}\text { Asses } \\
\text { Tools }\end{array}$ & & Comp & acies \\
\hline As a whole & - & $\begin{array}{l}\text { Mean } \\
\text { 3.39 }\end{array}$ & $\begin{array}{l}\text { VI } \\
\text { M }\end{array}$ & $\begin{array}{l}\text { Mean } \\
\mathbf{4 . 0 7}\end{array}$ & $\begin{array}{l}\text { VI } \\
\mathbf{H} \\
\end{array}$ & $\begin{array}{l}\text { Mean } \\
\text { 3.41 }\end{array}$ & $\begin{array}{l}\text { VI } \\
\mathbf{H} \\
\end{array}$ & $\begin{array}{l}\text { Mean } \\
\mathbf{3 . 0 4} \\
\end{array}$ & $\begin{array}{l}\text { VI } \\
\text { M } \\
\end{array}$ \\
\hline $\begin{array}{r}\text { Legend: } \mathrm{VI}= \\
\mathrm{VH} \\
\mathrm{H}\end{array}$ & $\begin{array}{l}\text { l Interpretation } \\
\text { ry High }\end{array}$ & & Mode & $\begin{array}{l}\mathrm{L}= \\
\mathrm{VL}\end{array}$ & ery & & & & \\
\hline
\end{tabular}

The level of difficulty of teachers towards Technology and Livelihood Education was moderate on physical facilities and competencies while high on instructional materials and assessment tools.

Table 3.2: Level of Difficulty of Students Grouped When Variables are taken as a Whole

\begin{tabular}{|c|c|c|c|c|c|c|c|c|c|}
\hline \multirow[t]{2}{*}{ Variable } & \multirow[t]{2}{*}{ Category } & \multicolumn{2}{|c|}{$\begin{array}{l}\text { Physical } \\
\text { Facilities }\end{array}$} & \multicolumn{2}{|c|}{$\begin{array}{l}\text { Instructional } \\
\text { Materials }\end{array}$} & \multicolumn{2}{|c|}{$\begin{array}{l}\text { Assessment } \\
\text { Tools }\end{array}$} & \multicolumn{2}{|c|}{ Competencies } \\
\hline & & Mean & VI & Mean & VI & Mean & VI & Mean & VI \\
\hline As a whole & - & 2.60 & $\mathbf{L}$ & 2.94 & M & 3.04 & $\mathbf{M}$ & 2.54 & $\mathbf{L}$ \\
\hline Legend: VI = & $\begin{array}{l}\text { Interpretation } \\
=\text { Very High }\end{array}$ & $\mathrm{H}=$ & & $M=$ & $\begin{array}{r}\mathrm{L} \\
\text { Moder }\end{array}$ & & & Very L & \\
\hline
\end{tabular}

The level of difficulty of students was low on physical facilities and competencies while moderate on instructional materials and assessment tools. 
Table 4.1: Extent of Coping Strategy of Teachers When Variables are taken as a Whole

\begin{tabular}{|c|c|c|c|c|c|c|c|c|c|}
\hline \multirow[t]{2}{*}{ Variable } & \multirow[t]{2}{*}{ Category } & \multicolumn{2}{|c|}{$\begin{array}{l}\text { Physical } \\
\text { Facilities }\end{array}$} & \multicolumn{2}{|c|}{$\begin{array}{l}\text { Instructional } \\
\text { Materials }\end{array}$} & \multicolumn{2}{|c|}{$\begin{array}{l}\text { Assessment } \\
\text { Tools }\end{array}$} & \multicolumn{2}{|c|}{ Competencies } \\
\hline & & Mean & VI & Mean & VI & Mean & VI & Mean & VI \\
\hline As a whole & - & 3.28 & $\mathbf{M}$ & 3.40 & $\mathbf{M}$ & 3.62 & G & 3.76 & G \\
\hline
\end{tabular}

Table 4.2: Extent of Coping Strategy of Students When Variables are taken as a Whole

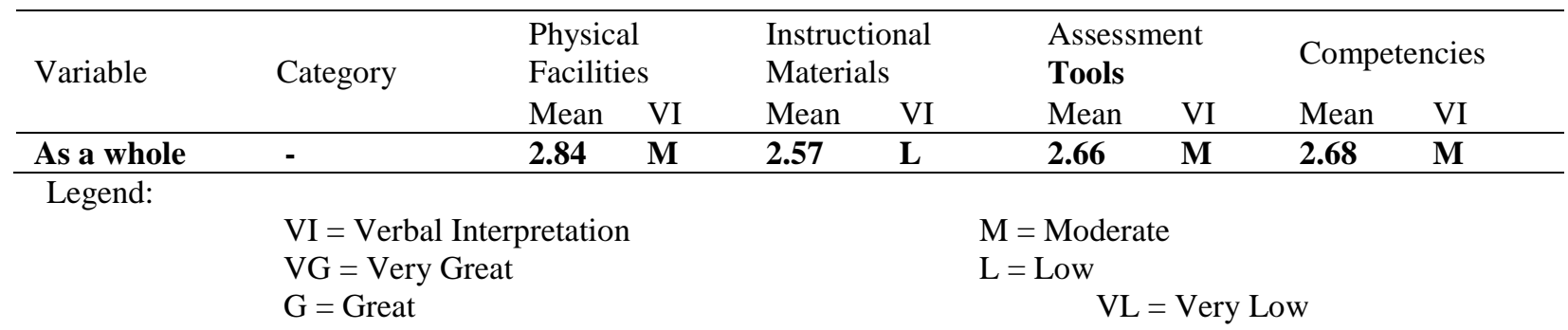

The students' extent of coping strategy was moderate on physical facilities, assessment tools, and competencies while low on instructional materials. Table 5.1: Difference in the Level of Difficulty of Teachers According to Age, Sex, Length of
Service, and Educational Qualification

\begin{tabular}{|c|c|c|c|c|c|c|c|c|}
\hline $\begin{array}{l}\text { Selected } \\
\text { Variables }\end{array}$ & Category & $\mathbf{N}$ & df & $\begin{array}{l}\text { Mea } \\
\text { n }\end{array}$ & $\begin{array}{l}\text { Mean } \\
\text { Difference }\end{array}$ & t-value & p-value & Interpretation \\
\hline \multirow{2}{*}{ Age } & Younger & 13 & 12 & 3.79 & \multirow{2}{*}{0.79} & \multirow[b]{2}{*}{2.977} & \multirow[b]{2}{*}{0.007} & \multirow[b]{2}{*}{ Significant } \\
\hline & Older & 13 & 12 & 3.00 & & & & \\
\hline \multirow{2}{*}{ Sex } & Male & 13 & 12 & 3.50 & \multirow{2}{*}{0.21} & \multirow{2}{*}{0.706} & \multirow{2}{*}{0.487} & \multirow{2}{*}{ Not Significant } \\
\hline & Female & 13 & 12 & 3.29 & & & & \\
\hline \multirow{2}{*}{$\begin{array}{l}\text { Length } \\
\text { Service }\end{array}$} & Shorter & 13 & 12 & 3.53 & \multirow{2}{*}{0.28} & \multirow{2}{*}{0.913} & \multirow{2}{*}{0.370} & \multirow{2}{*}{ Not Significan } \\
\hline & Longer & 13 & 12 & 3.25 & & & & \\
\hline \multirow{2}{*}{$\begin{array}{l}\text { Educational } \\
\text { Qualification }\end{array}$} & $\begin{array}{l}\text { Bachelor's } \\
\text { degree }\end{array}$ & 16 & 15 & 3.46 & \multirow{2}{*}{0.18} & \multirow{2}{*}{0.580} & \multirow{2}{*}{0.567} & \multirow{2}{*}{ Not Significan } \\
\hline & $\begin{array}{l}\text { Master's } \\
\text { degree }\end{array}$ & 10 & 9 & 3.28 & & & & \\
\hline
\end{tabular}

A significant difference existed in the level of difficulty on the use and provision of assessment tools when teachers were grouped according to age, except in the three areas.

Table 5.2: Difference in the Level of Difficulty of Teachers in the aforementioned Areas When Grouped According to Subject Taught

\begin{tabular}{lllllllll}
\hline \multirow{2}{*}{ Area } & $\begin{array}{l}\text { Sources } \\
\text { Variation }\end{array}$ & of & $\begin{array}{l}\text { Sum } \\
\text { Squares }\end{array}$ & of & $\begin{array}{l}\text { Mean } \\
\text { Square }\end{array}$ & F-value & p-value & Interpretation \\
\hline \multirow{2}{*}{ Physical Facilities } & Between Groups & 4.302 & 7 & 0.615 & & & \\
& Within Groups & 4.152 & 18 & 0.231 & 2.664 & 0.044 & Significant \\
& Total & 8.454 & 25 & & & & \\
Instructional & Between Groups & 4.660 & 7 & 0.666 & & & \\
Materials & Within Groups & 8.010 & 18 & 0.445 & 1.496 & 0.231 & \multirow{2}{*}{ Not Significant } \\
\hline
\end{tabular}




\begin{tabular}{llllllll}
\hline \multirow{2}{*}{ Assessment Tools } & Between Groups & 7.837 & 7 & 1.120 & & & \\
& Within Groups & 15.654 & 18 & 0.870 & 1.287 & 0.311 & Not Significant \\
& Total & 23.491 & 25 & & & & \\
Competencies & Between Groups & 6.941 & 7 & 0.992 & & & Not Significant \\
& Within Groups & 7.618 & 18 & 0.423 & 2.343 & 0.069 & \\
& Total & 14.559 & 25 & & & & \\
\hline
\end{tabular}

A significant difference also existed on physical facilities, except on the other three areas when teachers were grouped according to subject taught.

Table 5.3: Difference in the Level of Difficulty of Students in the Aforementioned Areas when Grouped According to Age

\begin{tabular}{lllllllll}
\hline Area & $\begin{array}{l}\text { Age } \\
\text { Category }\end{array}$ & $\mathbf{n}$ & $\mathbf{d f}$ & $\begin{array}{l}\text { Mea } \\
\mathbf{n}\end{array}$ & $\begin{array}{l}\text { Mean } \\
\text { Difference }\end{array}$ & t-value & p-value & Interpretation \\
\hline Physical & Younger & 137 & 136 & 3.43 & -0.06 & -0.945 & 0.345 & Not Significant \\
Facilities & Older & 177 & 176 & 3.49 & & & & \\
Instructional & Younger & 137 & 136 & 2.85 & -0.16 & -2.136 & 0.033 & Significant \\
Materials & Older & 177 & 176 & 3.01 & & & & Not Significant \\
Assessment & Younger & 137 & 136 & 3.04 & & -1.850 & 0.065 & \\
Tools & Older & 177 & 176 & 3.18 & -0.14 & & & Significant \\
Competencies & Younger & 137 & 136 & 3.02 & -0.22 & -2.606 & 0.010 & \\
\hline
\end{tabular}

There was a significant difference existed in the level of difficulty on instructional materials and competencies when students were grouped according to age.

Table 5.4: Difference in the Level of Difficulty of Students in the Aforementioned Areas when
Grouped According to Sex

\begin{tabular}{|c|c|c|c|c|c|c|c|c|}
\hline Area & $\begin{array}{l}\text { Sex } \\
\text { Category }\end{array}$ & $\mathbf{n}$ & Df & $\begin{array}{l}\text { Mea } \\
\text { n }\end{array}$ & $\begin{array}{l}\text { Mean } \\
\text { Difference }\end{array}$ & t-value & p-value & Interpretation \\
\hline \multirow{2}{*}{$\begin{array}{l}\text { Physical } \\
\text { Facilities }\end{array}$} & Male & 155 & 154 & 3.44 & \multirow{2}{*}{-0.05} & \multirow{2}{*}{-0.784} & \multirow{2}{*}{0.434} & \multirow{2}{*}{ Not Significant } \\
\hline & Female & 158 & 157 & 3.49 & & & & \\
\hline \multirow{2}{*}{$\begin{array}{l}\text { Instructional } \\
\text { Materials }\end{array}$} & Male & 155 & 154 & 2.90 & \multirow{2}{*}{-0.08} & \multirow{2}{*}{-0.979} & \multirow{2}{*}{0.328} & \multirow{2}{*}{ Not Significan } \\
\hline & Female & 158 & 157 & 2.98 & & & & \\
\hline \multirow{2}{*}{$\begin{array}{l}\text { Assessment } \\
\text { Tools }\end{array}$} & Male & 155 & 154 & 3.12 & \multirow{2}{*}{0.00} & \multirow{2}{*}{0.031} & \multirow{2}{*}{0.975} & \multirow{2}{*}{ Not Significant } \\
\hline & Female & 158 & 157 & 3.12 & & & & \\
\hline \multirow{2}{*}{ Competencies } & Male & 155 & 154 & 3.16 & \multirow{2}{*}{0.03} & \multirow{2}{*}{0.346} & \multirow{2}{*}{0.729} & \multirow{2}{*}{ Not Significant } \\
\hline & Female & 158 & 157 & 3.13 & & & & \\
\hline
\end{tabular}

When students were grouped according to sex, the level of significance revealed no significant difference existed in the level of their difficulty in the four areas.

Table 5.5: Difference in the Level of Difficulty of Students in the Aforementioned Areas when Grouped According to Year Level

\begin{tabular}{lllllllll}
\hline Area & Category & $\mathbf{n}$ & $\mathbf{d f}$ & $\begin{array}{l}\text { Mea } \\
\mathbf{n}\end{array}$ & $\begin{array}{l}\text { Mean } \\
\text { Difference }\end{array}$ & t-value & p-value & Interpretation \\
\hline Physical & Grade 9 & 167 & 166 & 2.83 & & & & Significant \\
$\begin{array}{l}\text { Facilities } \\
\text { Instructional }\end{array}$ & Grade 10 & 146 & 145 & 3.13 & -0.30 & -3.242 & 0.001 & Not Significant \\
Materials & Grade 9 & 167 & 166 & 3.29 & & & & \\
Assessment & Grade 10 & 146 & 145 & 3.44 & -0.15 & -1.591 & 0.113 & \\
Tools & Grade 9 & 167 & 166 & 3.39 & -0.18 & -2.413 & 0.016 & Significant \\
\hline
\end{tabular}




\begin{tabular}{lllllllll}
\hline \multirow{2}{*}{ Competencies } & Grade 9 & 167 & 166 & 2.91 & \multirow{2}{*}{0.00} & \multirow{2}{*}{0.005} & 0.996 & \multirow{2}{*}{ Not Significant } \\
& Grade 10 & 146 & 145 & 2.91 & & &
\end{tabular}

Also a significant difference existed on physical facilities and assessment tools when they were grouped according to year level.

\section{Table 5.6: Difference in the Level of Difficulty of Students in the Aforementioned Areas when Grouped According to Specialization}

\begin{tabular}{|c|c|c|c|c|c|c|c|}
\hline Area & $\begin{array}{ll}\text { Sources } \\
\text { Variation }\end{array}$ & $\begin{array}{l}\text { Sum of } \\
\text { Squares }\end{array}$ & df & $\begin{array}{l}\text { Mean } \\
\text { Square }\end{array}$ & F-value & p-value & Interpretation \\
\hline \multirow{3}{*}{ Physical Facilities } & Between Groups & 10.531 & 7 & 1.504 & & & \\
\hline & Within Groups & 103.973 & 305 & 0.341 & 4.413 & 0.000 & Significant \\
\hline & Total & 114.504 & 312 & & & & \\
\hline \multirow{3}{*}{$\begin{array}{l}\text { Instructional } \\
\text { Materials }\end{array}$} & Between Groups & 6.870 & 7 & 0.981 & & & \\
\hline & Within Groups & 140.055 & 305 & 0.459 & 2.137 & 0.040 & Significant \\
\hline & Total & 146.924 & 312 & & & & \\
\hline \multirow{3}{*}{ Assessment Tools } & Between Groups & 4.552 & 7 & 0.650 & & & \\
\hline & Within Groups & 132.023 & 305 & 0.433 & 1.502 & 0.166 & Not Significant \\
\hline & Total & 136.575 & 312 & & & & \\
\hline \multirow{3}{*}{ Competencies } & Between Groups & 11.498 & 7 & 1.643 & & & \\
\hline & Within Groups & 166.718 & 305 & 0.547 & 3.005 & 0.005 & Significant \\
\hline & Total & 178.217 & 312 & & & & \\
\hline
\end{tabular}

Significant difference existed in the areas of physical facilities, instructional materials, and competencies except on assessment tools when students were grouped according to area of specialization.

\section{Table 6.1: Difference in the Extent of Coping of Teachers According to Age, Sex, Length of Service, and Educational Qualification}

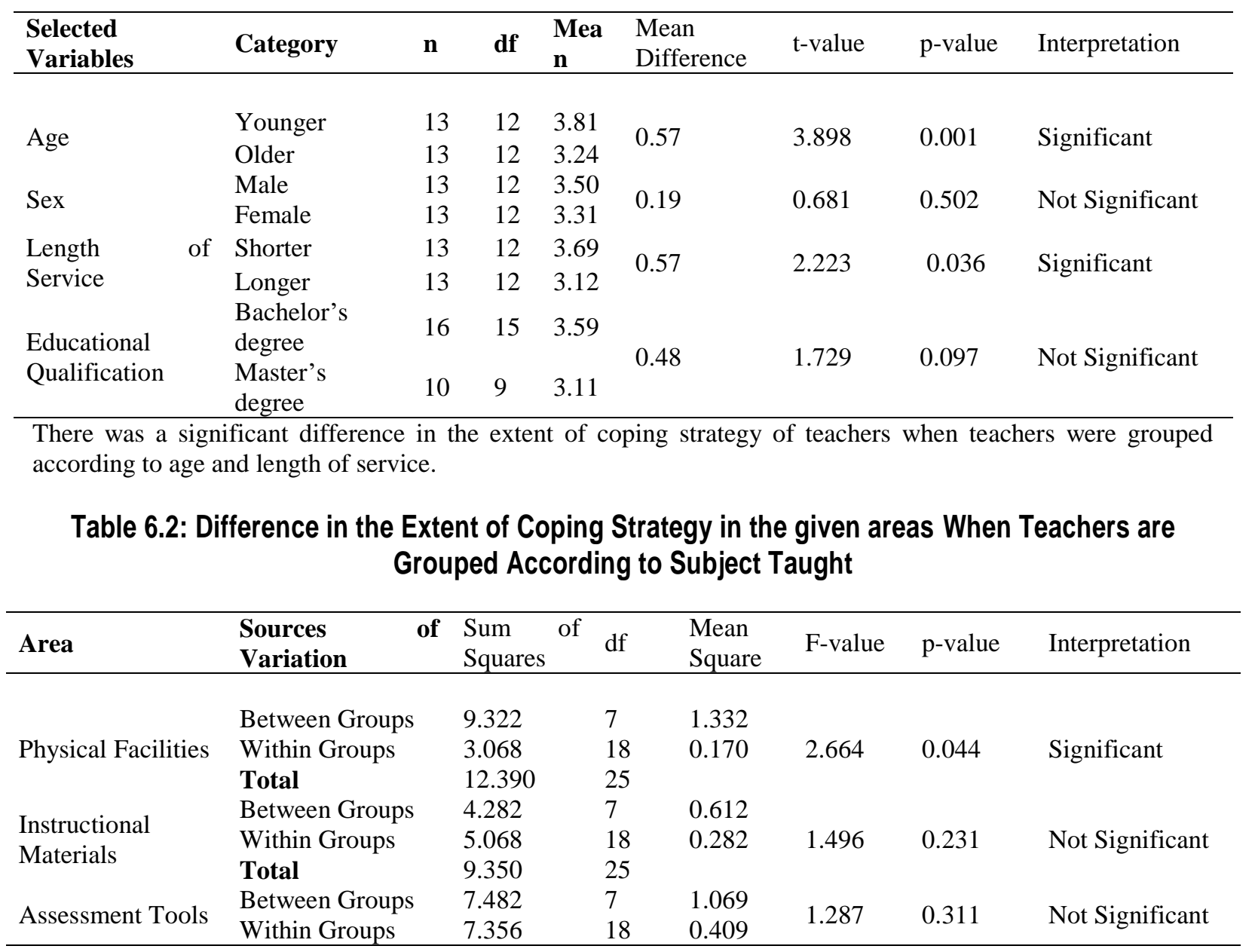




\begin{tabular}{|c|c|c|c|c|c|c|c|}
\hline \multirow{4}{*}{ Competencies } & Total & 14.838 & 25 & & & & \multirow{4}{*}{ Not Significant } \\
\hline & Between Groups & 13.005 & 7 & 1.858 & & & \\
\hline & Within Groups & 21.466 & 18 & 1.193 & 2.343 & 0.069 & \\
\hline & Total & 34.471 & 25 & & & & \\
\hline
\end{tabular}

Likewise, a significant difference existed on physical facilities when teachers were grouped according to subject taught.

Table 6.3: Difference in the Extent of Coping Strategy of Students in the given Areas when Grouped According to Age

\begin{tabular}{|c|c|c|c|c|c|c|c|c|}
\hline Area & $\begin{array}{l}\text { Age } \\
\text { Category }\end{array}$ & $\mathrm{n}$ & $\mathrm{df}$ & $\begin{array}{l}\text { Mea } \\
\mathrm{n} \\
\end{array}$ & $\begin{array}{l}\text { Mean } \\
\text { Difference } \\
\end{array}$ & t-value & p-value & Interpretation \\
\hline \multirow{2}{*}{$\begin{array}{l}\text { Physical } \\
\text { Facilities }\end{array}$} & Younger & 137 & 136 & 2.94 & \multirow{2}{*}{-0.06} & \multirow{2}{*}{-0.685} & \multirow{2}{*}{0.494} & \multirow{2}{*}{ Not Significant } \\
\hline & Older & 177 & 176 & 3.00 & & & & \\
\hline \multirow{2}{*}{$\begin{array}{l}\text { Instructional } \\
\text { Materials }\end{array}$} & Younger & 137 & 136 & 3.37 & \multirow{2}{*}{0.01} & \multirow{2}{*}{0.115} & \multirow{2}{*}{0.908} & \multirow{2}{*}{ Not Significant } \\
\hline & Older & 177 & 176 & 3.36 & & & & \\
\hline \multirow{2}{*}{$\begin{array}{l}\text { Assessment } \\
\text { Tools }\end{array}$} & Younger & 137 & 136 & 3.43 & \multirow{2}{*}{-0.07} & \multirow{2}{*}{-0.907} & \multirow{2}{*}{0.365} & \multirow{2}{*}{ Not Significant } \\
\hline & Older & 177 & 176 & 3.50 & & & & \\
\hline \multirow{2}{*}{ Competencies } & Younger & 137 & 136 & 2.93 & \multirow{2}{*}{0.04} & \multirow{2}{*}{0.441} & \multirow{2}{*}{0.659} & \multirow{2}{*}{ Not Significant } \\
\hline & Older & 177 & 176 & 2.89 & & & & \\
\hline
\end{tabular}

The data displayed no significant difference existed in the extent of coping strategy of students when grouped according to age across the four areas.

Table 6.4: Difference in the Extent of Coping Strategy of Students in the given areas when Grouped According to Sex

\begin{tabular}{|c|c|c|c|c|c|c|c|c|}
\hline Area & $\begin{array}{l}\text { Sex } \\
\text { Category } \\
\end{array}$ & $\mathbf{n}$ & df & $\begin{array}{l}\text { Mea } \\
\text { n } \\
\end{array}$ & $\begin{array}{l}\text { Mean } \\
\text { Difference }\end{array}$ & t-value & p-value & Interpretation \\
\hline \multirow{2}{*}{$\begin{array}{l}\text { Physical } \\
\text { Facilities }\end{array}$} & Male & 155 & 154 & 2.98 & \multirow{2}{*}{0.02} & \multirow{2}{*}{0.297} & \multirow{2}{*}{0.767} & \multirow{2}{*}{ Not Significant } \\
\hline & Female & 158 & 157 & 2.96 & & & & \\
\hline \multirow{2}{*}{$\begin{array}{l}\text { Instructional } \\
\text { Materials }\end{array}$} & Male & 155 & 154 & 3.33 & \multirow{2}{*}{-0.56} & \multirow{2}{*}{-0.631} & \multirow{2}{*}{0.529} & \multirow{2}{*}{ Not Significant } \\
\hline & Female & 158 & 157 & 3.89 & & & & \\
\hline \multirow{2}{*}{$\begin{array}{l}\text { Assessment } \\
\text { Tools }\end{array}$} & Male & 155 & 154 & 3.47 & \multirow{2}{*}{0.00} & \multirow{2}{*}{-0.049} & \multirow{2}{*}{0.961} & \multirow{2}{*}{ Not Significant } \\
\hline & Female & 158 & 157 & 3.47 & & & & \\
\hline \multirow{2}{*}{ Competencies } & Male & 155 & 154 & 2.85 & \multirow{2}{*}{-0.11} & \multirow{2}{*}{-1.316} & \multirow{2}{*}{0.189} & \multirow{2}{*}{ Not Significant } \\
\hline & Female & 158 & 157 & 2.96 & & & & \\
\hline
\end{tabular}

When students were grouped according to sex, the data revealed no significant difference existed in the extent of their coping strategy in all four areas.

Table 6.5: Difference in the Extent of Coping Strategy of Students in the given Areas when Grouped According to Year Level

\begin{tabular}{lllllllll}
\hline Area & Category & $\mathbf{n}$ & $\mathbf{d f}$ & $\begin{array}{l}\text { Mea } \\
\mathbf{n}\end{array}$ & $\begin{array}{l}\text { Mean } \\
\text { Difference }\end{array}$ & t-value & p-value & Interpretation \\
\hline Physical & Grade 9 & 167 & 166 & 3.45 & & & & \\
Facilities & Grade 10 & 146 & 145 & 3.49 & -0.04 & -0.618 & 0.537 & Not Significant \\
Instructional & Grade 9 & 167 & 166 & 2.86 & -0.17 & -2.154 & 0.032 & Significant \\
Materials & Grade 10 & 146 & 145 & 3.03 & & & & Significant \\
Assessment & Grade 9 & 167 & 166 & 3.01 & -0.23 & -3.034 & 0.003 & \\
\hline
\end{tabular}




\begin{tabular}{lllllllll}
\hline Tools & Grade 10 & 146 & 145 & 3.24 & & & & \\
\multirow{2}{*}{ Competencies } & Grade 9 & 167 & 166 & 2.99 & -0.34 & -4.027 & 0.004 & Significant \\
& Grade 10 & 146 & 145 & 3.33 & & & & \\
\hline
\end{tabular}

There was a significant difference in the coping strategy of students on instructional materials, assessment tools, and competencies when they were grouped according to year level.

\section{Table 6.6: Difference in the Extent of Coping Strategy of Students in the given areas when Grouped According to Specialization}

\begin{tabular}{|c|c|c|c|c|c|c|c|}
\hline Area & $\begin{array}{l}\text { Sources } \\
\text { Variation }\end{array}$ & $\begin{array}{l}\text { Sum of } \\
\text { Squares }\end{array}$ & df & $\begin{array}{l}\text { Mean } \\
\text { Square }\end{array}$ & F-value & $\mathrm{p}$-value & Interpretation \\
\hline \multirow{3}{*}{ Physical Facilities } & Between Groups & 25.142 & 7 & 3.592 & & & \\
\hline & Within Groups & 174.539 & 305 & 0.572 & 6.276 & 0.000 & Significant \\
\hline & Total & 199.682 & 312 & & & & \\
\hline \multirow{3}{*}{$\begin{array}{l}\text { Instructional } \\
\text { Materials }\end{array}$} & Between Groups & 38.098 & 7 & 5.443 & & & \\
\hline & Within Groups & 152.575 & 305 & 0.500 & 10.880 & 0.000 & Significant \\
\hline & Total & 190.673 & 312 & & & & \\
\hline \multirow{3}{*}{ Assessment Tools } & Between Groups & 14.938 & 7 & 2.134 & & & \\
\hline & Within Groups & 124.510 & 305 & 0.408 & 5.227 & 0.000 & Significant \\
\hline & Total & 139.448 & 312 & & & & \\
\hline \multirow{3}{*}{ Competencies } & Between Groups & 17.880 & 7 & 2.554 & & & \\
\hline & Within Groups & 172.767 & 305 & 0.566 & 4.509 & 0.000 & Significant \\
\hline & Total & 190.647 & 312 & & & & \\
\hline
\end{tabular}

Likewise, there was a significant difference in all four areas-namely, physical facilities, instructional materials, assessment tools, and competencies when the students were grouped according to specialization.

\section{Conclusion}

On the bases of the foregoing findings of the study, the researcher arrived at the following conclusions:

There was inadequacy of instructional materials which are the primary medium for the delivery of the Technology and Livelihood Education. In effect, the teachers' competencies are affected for not being able to connect the subject area content to real life applications. With this, valued learning outcomes were also hampered.

The available facilities, materials, and tools conform to the recommended numbers which are required to support the students' needs such as improving their competencies in the Technology and Livelihood Education subject.

Due to inadequacy of assessment tools, Technology and Livelihood Education teachers exerted a great deal of effort in looking for related materials.

The students were a bit adjusted to the environmental components of Technology and
Livelihood Education classes; hence, they still have to cope in moderate extent in getting the most out of the Technology and Livelihood Education lessons.

Generally, teachers' difficulties in teaching Technology and Livelihood Education cannot be said to exist due to problems associated with their grouping, except their age and subject taught, but to indicators relative to physical facilities or those things that are essentially needed for effective teaching and learning process to take place.

Difficulties in learning Technology and Livelihood Education could not be attributed to factors which are personal in nature but to matters relative to instructional materials.

The inadequacy of the provision of the needed assessment tools necessitates the young and inexperienced teachers to find ways to properly measure how well their students have understood their lessons.

Teachers teaching different subjects needed to deal to some extent on these inadequacies for them to discharge their functions well and help students learn effectively.

The extent of coping strategy of the students essentially differed due to the fact that each year 
level and specialization has distinct difficulties, problems, and needs.

\section{Recommendations}

In light of the findings and conclusions of the study, the following recommendations are advanced for potential improvements:

The school officials should provide additional instructional materials such as textbooks, modules, worksheets among others for teachers and students' use.

The school officials should provide additional assessment tools particularly laboratory rooms and tools to match the increasing number of users (i.e. teachers and students alike).

The school officials should provide the students with adequate classrooms equipped with proper lighting and ventilation to facilitate quality learning.

The school officials should sufficiently provide the newly hired and inexperienced teachers with necessary instructional and assessment tools to help them deliver their Technology Livelihood Education lessons well. Likewise, they should be sent to training to enhance their teaching skills.

The teachers should continue to upgrade themselves through attending related seminars and trainings to further enhance their teaching skills.

The young and inexperienced teachers should be sent to training to make them equip with the right knowledge and skills in teaching Technology and Livelihood Education.

The students can be provided trainings on coping strategies to address their individual problems and needs.

The students can be provided with proper orientation program so that they may be able to understand the underlying strengths and weaknesses on the implementation of the TLE programs.

Finally, the proposed enhancement program should be implemented to reinforce the teachers' teaching skills and students' learning capabilities.

\section{Conflict of Interest}

There is no Conflict of Interest in this work.

\section{References}

1. American Psychological Association, (2011). The Guidelines for Psychological Practice. Adopted by the APA Council of
Representatives, February 18-20, 2011, The Guidelines are available on the APA website at http://www.apa.org/pi/lgbt/resources/guideline s.aspx; accessed 12/30/2016.

2. Agarwal, Nidhi, (2007). A Matter of Styles in Education. Asian Journal of Psychology \& Education, 69(5).

3. Agarwal, Nidhi, (2018). A Study on Content Generation for Internet Usage. International Journal of Advanced Research and Development, 3(2); 1380-1382.

4. Bhattacherjee, A., (2012). Social Science Research: Principles, Methods, and Practices. University of South Florida, U.S.A.

5. Calderon, J., (2012). Methods of Research and Thesis Writing. National Book Store, Quad Alpha Centrum Building, 125 Pioneer Street, Mandaluyong City, 1550 Philippines.

6. Chaaban, Y. and Moloney, R., (2016). An Exploratory Study of the Factors Associated With Literacy Teachers' Integration of Technology: A Study of Lebanese Schools. Qatar University.

7. Chang, I.H., (2012). The Effect of Principals' Technological Leadership on Teachers' Technological Literacy and Teaching Effectiveness in Taiwanese Elementary Schools. Educational Technology \& Society, 15 (2), 328-340.

8. Chow, P., (2015). Teacher's Attitudes Towards Technology in the Classroom. Department of Curriculum, Teaching and Learning Ontario Institute for Studies in Education of the University of Toronto, Canada.

9. Cristobal, A. and Cristobal, M.C., (2013). Research Made Easier: A Step-by-Step Process. C \& E Publishing, Inc. 839 EDSA, South Triangle, Quezon City, Philippines.

10. Deauna, M., (2011). Applied Educational Statistics. C \& E Publishing, Inc., 839 EDSA, South Triangle, Quezon City, Philippines.

11. Department of Education Order No. 37, (2003). DepEd Complex, Meralco Avenue, Pasig City, Metro Manila, Philippines.

12. Department of Education Memorandum No. 468, (2008). DepEd Complex, Meralco Avenue, Pasig City, Metro Manila, Philippines.

13. Department of Education Memorandum No. 399, (2009). DepEd Complex, Meralco Avenue, Pasig City, Metro Manila, Philippines.

14. Department of Education Order No. 76, (2010). DepEd Complex, Meralco Avenue, Pasig City, Metro Manila, Philippines.

15. Department of Education Order No. 67, (2012). Enclosure No. 2. DepEd Complex, Meralco Avenue, Pasig City, Metro Manila, Philippines. 
16. De Belen, R. and Feliciano, J., (2015). Basic Statistics for Research. First Edition, Wiseman's Books Trading, Inc., Philippines.

17. Garcia,Y., et al, (2014). Difficulties in Reading Comprehension and Metacognitive Strategies for Technology Livelihood Education Students. College of Education Mindanao State University-Iligan Institute of Technology, Philippines.

18. Gravetter, F. and Walnau, L., (2012). Statistics for Behavioral Sciences. Cengage Learning Asia Pte. Ltd., 5 Shenton Way \#01-01 UIC Building, Singapore.

19. Guiner, D., (2013). Competencies of Technology and Livelihood Education (TLE)Instructors: Input to a Training Module in Industrial Arts. Don Mariano Marcos Memorial State University Bacnotan, La Union, Philippines.

https://stats.oecd.org/glossary/detail.asp?ID=7 44; accessed 12/30/2016

http://www.simplypsychology.org/attitudes.ht $\mathrm{ml}$; retrieved 12/30/16

http://study.com/academy/lesson/copingstrategy-definition-lesson-quiz.html; retrieved $12 / 30 / 16$ http://study.com/academy/lesson/instructionalmaterials-definition-examples-evaluation.html; retrieved 01/10/2017

20. Lazarus, R.S. \& Folkman, S., (1984). Stress, Appraisal and Coping. New York: Springer.

21. Ronald John C. Sayson, Enna S. Bodoso, (2019). Basic English Skills Training (BEST) E-Learning Tool: CHMSC Experience, Globus An Internatinal Journal of Management \& IT, 10(2); 44-50, ISSN: 0975-721X.

22. Retome, et al, (2012). Instructional Assessment of Technology and Livelihood Education (TLE) Program. Education Department, Southern Leyte State University San Juan Campus, 6611 San Juan, Southern Leyte, Philippines.

23. Salwa, Saeed Al-Harbi, (2019). The Influence of Media in Children's Language Development. Globus Journal of Progressive Education, $\quad 9(2) ; \quad 23-27, \quad$ doi: 10.46360/globus.9.2.2020.23-27.

24. Sweller, J., (1988). Cognitive Load Theory, Learning Difficulty, and Instructional Design. University of NSW, Sydney, Australia.

25. Vygotsky, M.E., (1973). Understanding the Learning theory, New York: Rutledge Publishers.

How to cite this article:

Laganhon, Aljaven Bruce C. (2020). Teachers and Students' Difficulty and Coping Strategy Towards Technology and Livelihood Education Program. Globus Journal of Progressive Education, 10(1): 82-91. 